PROCEEDINGS OF THE

AMERICAN MATHEMATICAL SOCIETY

Volume 100, Number 1, May 1987

\title{
A NOTE ON PUSHING UP
}

\author{
PAUL S. FAN
}

\begin{abstract}
The amalgam method is used to handle some so-called pushingup problems in finite groups.
\end{abstract}

1. Introduction. Generally speaking, pushing-up problems in finite group theory have been posed in terms of obstructions:

Suppose $S \in \operatorname{Syl}_{p}(P)$ such that no characteristic subgroup of $S$ is normal in $P$. What then can we say about $S$ ? In Goldschmidt's fundamental paper [G], he introduced what is now called the amalgam method. This was later in particular applied to a pushing-up problem by Stellmacher $[\mathbf{S}]$ that simplified the works of Niles $[\mathbf{N}]$ and Baumann $[\mathbf{B}]$. So let us reformulate our pushing-up question in terms of amalgams. (See $[\mathbf{G}]$ for the basic definitions.)

Suppose $P$ and $H$ are abstract finite groups. We say $P \supseteq S \subseteq H$ is a pushing-up amalgam for $P / O_{p}(P)$ if

(1) $S \in \operatorname{Syl}_{p}(P)$.

(2) No nontrivial subgroup of $S$ is normal in $P$ and $H$.

(3) $S \unlhd H$.

Take $X$ to be a finite group and $S \in \operatorname{Syl}_{p}(X)$. Notice that if $M$ is a maximal $p$-local but not a $p$-parabolic of $X$ (i.e., $N_{X}(S) \nsubseteq M$ ) then one clearly has a pushing-up amalgam. Or if $N_{X}(S)$ is contained in a unique maximal $p$-local $M$ of $X$, then one also has a pushing-up amalgam, unless of course $M$ is strongly embedded. We further remark that there is some motivation in changing (1) to require simply that $S$ be a $p$-group containing $O_{p}(P)$. (See $[\mathbf{G n}$, p. 280] and $[\mathbf{C}]$.) This actually does not entail any essential changes in the argument of our theorem below.

So as a general goal, given any pushing-up amalgam for a $\operatorname{chev}(p)$-group one would like to be able to describe $S$. In this note we give a small indication to be optimistic about solving this problem in general. (See also $[\mathbf{M}]$.) Specifically, we consider pushing-up amalgams for $\operatorname{chev}(p)$-groups satisfying Hypothesis A below. First, we define a $\operatorname{GF}(p) X$-module $W$ to be an FF-module if $C_{X}(W)$ is a $p^{\prime}$-group and $X$ has a nontrivial elementary abelian p-group $A$ such that $\left|W: C_{W}(A)\right| \leq|A|$. $A$ is called an offending subgroup.

HYPOTHESIS A. $X$ is a group where $X / \phi(X)$ is simple. For any irreducible FF, $\operatorname{GF}(p) X$-module $W$ and any quadratic offending subgroup $A$ of $X$, we have

(1) $\left|W: C_{W}(A)\right|=|A|$.

(2) $A$ is a maximal quadratic subgroup of $X$.

(3) If $A \subseteq S \in \operatorname{Syl}_{p}(X)$, then there exist $g \in X$ such that $\left\langle A, S^{g}\right\rangle=X$ and whenever $A \Varangle S,\left\langle A,\left\langle A^{S g}\right\rangle\right\rangle S=X$.

Received by the editors November 5, 1985 and, in revised form, February 1, 1986. 1980 Mathematics Subject Classification (1985 Revision). Primary 20D05, $20 \mathrm{~B} 05$. 
By Lemma (2.3) in [T] and Lemma (2.1) in [S] we see that $U_{4}(q), U_{5}(q), G_{2}(q)$, $q$ even, $\hat{A}_{6}$, and $\mathrm{SL}_{2}(q)$ satisfy Hypothesis A.

To state our result, we require some standard definitions from the amalgam method. (For the details see $[\mathbf{G}]$ and $\S 2$ below.) Given the amalgam $P \supseteq S \subseteq H$, $G=P *_{S} H$ acts faithfully on the completion graph $\Gamma=\Gamma(G ; P, H)$, and there exist adjacent vertices $\alpha$ and $\beta$ in $\Gamma$ such that $G_{\alpha}=P, G_{\beta}=H$ and $G_{\alpha, \beta}=S$. We set

$$
Z_{\alpha}=\left\langle\Omega_{1}(Z(S)): S \in \operatorname{Syl}_{p}\left(G_{\alpha}\right)\right\rangle
$$

and let $b=\min \left\{d(\lambda, \alpha): Z_{\alpha} \nsubseteq G_{\lambda}^{(1)}\right\}$.

THEOREM. If $P \supseteq S \subseteq H$ is a pushing-up amalgam for a group satisfying Hypothesis $\mathrm{A}$, then $b \leq 4$.

The point of course is that, in general, $b \leq 4$ along with the group $P / O_{p}(P)$ always yield a strong bound on the number of noncentral $p$-chief factors of $P$ in $O_{p}(P)$. To see the reason below, it is advisable to consult $\S 2$ for some definitions. Take $(\alpha, \alpha+1, \ldots, \alpha+5) \subseteq \Gamma$ where $(\alpha, \alpha+4)$ is a critical pair and let $G_{\alpha(i)}=$ $\bigcap_{x \in G_{\alpha}} G_{\alpha, \alpha+i}^{x}$. We then see from (3.2.2) and (3.1.4) that $\left\langle Z_{\alpha+4}^{G_{\alpha}}\right\rangle O_{p}\left(G_{x}\right)=G_{\alpha}$ and that $\left\langle Z_{\alpha+4}^{G_{\alpha}}\right\rangle$ centralizes $G_{\alpha(5)}$. Hence the noncentral $p$-chief factors are all contained in $O_{p}\left(G_{\alpha}\right) / G_{\alpha(4)}=G_{\alpha(2)} / G_{\alpha(4)}$ and $G_{\alpha(4)} / G_{\alpha(5)}$. We now observe that $\left|O_{p}\left(G_{\alpha}\right) / G_{\alpha(5)}\right|$ depends only on $\left|G_{\alpha} / O_{p}\left(G_{\alpha}\right)\right|$, since $G_{\alpha, \alpha+j} \subseteq G_{\alpha+j}^{(1)}$ for $j$ odd. Furthermore, for each pushing-up group $G_{\alpha} / O_{p}\left(G_{\alpha}\right)$, one should then be able to calculate the detail structure of $S$ as is done for example in $[\mathbf{S}$ and $\mathbf{M}$ ].

One final remark is that our use of the various parts of Hypothesis A is highly modular. Thus if one part of Hypothesis A is replaced by a weaker assumption, then this only entails a change in a very specific part of our argument.

2. Lemmas and notations. Take $P, H, S, G=P *_{S} H$, and the tree $\Gamma$ to be as in $\S 1$. We let $\alpha, \beta$ be adjacent vertices in $\Gamma$ such that $G_{\alpha}=P, G_{\beta}=H$ and $G_{\alpha \beta}=S$. A list of graph-theoretic notations follows.

(1) $\delta_{1} \sim \delta_{2}$ if $\delta_{1}, \delta_{2}$ are adjacent vertices in $\Gamma$.

(2) $\delta_{1} \equiv \delta_{2}$ if $d\left(\delta_{1}, \delta_{2}\right)$ is even, where $d($, $)$ is the usual distance metric on $\Gamma$.

(3) $\Delta(\delta)=\{\lambda \in \Gamma: \lambda \sim \delta\}$.

(4) Given a vertex $\lambda$ on a path $l$ in $\Gamma$, we write $\lambda+i$ or $\lambda-i(i \in \mathbf{Z})$ to mean the obvious vertices on $l$ distance $i$ from $\lambda$.

(5) $G_{\delta}^{(i)}=\bigcap_{d(\lambda, \delta) \leq i} G_{\lambda}$.

(6) For $\mu \not \equiv \alpha$, we let $S_{\mu}=G_{\mu, \varepsilon}$ for any $\varepsilon \sim \mu$.

(7) For any $\delta \equiv \alpha$, we set $Z_{\delta}=\left\langle\Omega_{1} Z(S): S \in \operatorname{Syl}_{p}\left(G_{\delta}\right)\right\rangle$.

(8) $b=\min \left\{d(\lambda, \alpha): Z_{\alpha} \nsubseteq G_{\lambda}^{(1)}\right\}$.

(9) We say $\left(\delta, \delta^{\prime}\right)$, where $\delta \equiv \alpha$, is a critical pair if $Z_{\delta} \nsubseteq G_{\delta}^{(1)}$ and $d\left(\delta, \delta^{\prime}\right)=b$.

In the lemmas below, $X$ is a finite group and $S \in \operatorname{Syl}_{p}(X)$.

(2.1) If $V$ is a $K X$-module where char $K=p$ and $V=\left\langle C_{V}(S)^{X}\right\rangle$, then $V=$ $[V, X] C_{V}(X)$.

ProOF. See (1.1) in $[\mathbf{M}]$. 
(2.2) Suppose $V$ is an $X$-module, $O_{p}(X)=1$ and $O^{p}(X / \phi(X))$ is minimal normal in $X / \phi(X)$. Then either $C_{X}(V) \subseteq \phi(X)$ or $C_{V}(S) \subseteq C_{V}(X)$.

Proof. Set $L$ to be the inverse image of $O^{p}(X / \phi(X))$ in $X$ and $C=C_{X}(V)$. Then we claim that either $C \subseteq L$ or $L \subseteq C$. For otherwise, we take $T \in \operatorname{Syl}_{p}(C)$ and observe that by Frattini's argument,

$$
X=C N_{X}(T)=\left(C_{L}(V) T\right) N_{X}(T)=N_{X}(T),
$$

which violates our hypothesis. Now we note that our claim easily implies the desired conclusion.

(2.3) Let $\bar{X}=X / O_{p}(X)$ and let $\bar{X} / \phi(\bar{X})$ be simple. Let $V=\left\langle C_{V}(S)^{X}\right\rangle$ be an $\mathrm{FF} \mathrm{GF}(p) \bar{X}$-module where $\bar{A} \subseteq \bar{X}$ is an offending subgroup. Suppose for all nontrivial irreducible $\mathrm{FF} \mathrm{GF}(p) \bar{X}$-modules $W,\left|W: C_{W}(\bar{A})\right|>|\bar{A}|^{1 / 2}$. Then $V / C_{V}(X)$ is an irreducible $\mathrm{FF} \mathrm{GF}(p) \bar{X}$-module.

Proof. Since $\phi(\bar{X})$ is a $p^{\prime}$-group, it follows from (2.2) that any noncentral irreducible factor module in $V$ is an FF $\bar{X}$-module. Hence by our hypothesis, $V$ contains a unique noncentral irreducible factor module. Therefore (2.1) yields that $V / C_{V}(X)$ is an irreducible $\bar{X}$-module.

(2.4). Let $Z=\left\langle\Omega_{1}(Z(S))^{X}\right\rangle$. Suppose $T \leq S$ and $Y \leq X$ such that $\left\langle T^{Y}\right\rangle$ acts transitively on $\left\{\Omega_{1}(Z(S))^{X}\right\}$ and $Y$ normalizes $[Z, T]$. Then $[[Z, T], T]=[Z, T]$.

Proof. Let $L=\left\langle T^{Y}\right\rangle$. Then we observe that

$$
Z=[Z, L] \Omega_{1}(Z(S)) .
$$

Since $\langle T, Y\rangle$ clearly normalizes $\left[Z, T^{y}\right]$ for all $y \in Y$, we obtain $\left[Z,\left\langle T^{Y}\right\rangle\right]=\left\langle\left[Z, T^{y}\right]\right.$ : $y \in Y\rangle=[Z, T]$. Hence $[Z, T]=[[Z, T], T]$.

3. Proof of Theorem. We use the notation given in $\S 2$. Our hypothesis here is that $G_{\alpha} \supseteq G_{\alpha \beta} \subseteq G_{\beta}, \alpha \sim \beta$, is a pushing-up amalgam for $\bar{G}_{\alpha}=G_{\alpha} / O_{p}\left(G_{\alpha}\right)$ and $\bar{G}_{\alpha}$ is a group satisfying Hypothesis A. Further, we might as well assume that $B \geq 1$.

(3.1) The following hold.

(1) $G_{\delta}$ acts transitively on $\Delta(\delta)$, for all $\delta \in \Gamma$.

(2) $G_{\alpha \beta}=G_{\beta}^{(1)}$ and $O_{p}\left(G_{\alpha}\right)=G_{\alpha}^{(1)}=G_{\alpha}^{(2)}$.

(3) $Z_{\alpha} \neq \Omega_{1}(Z(S))$ where $S \in \operatorname{Syl}_{p}\left(G_{\alpha}\right)$.

(4) $Z_{\alpha} \subseteq Z\left(O_{p}\left(G_{\alpha}\right)\right)$.

(5) $b$ is even.

(6) $O_{p}\left(G_{\alpha}\right) \in \operatorname{Syl}_{p}\left(C_{G_{\alpha}}\left(Z_{\alpha}\right)\right)$.

(7) For any p-group $T \subseteq G_{\alpha}$, we have $\left[T, Z_{\alpha}\right]=1$ if and only if $t \subseteq G_{\alpha}^{(2)}$.

Proof. (1), (2), and (3) are immediate consequences of the definition of a pushing-up amalgam. (4) follows from the fact that $b \geq 1$, and (5) is implied by (2).

Since $Z_{\alpha}=\left\langle C_{Z_{\alpha}}(\bar{S}): S \in \operatorname{Syl}_{p}\left(G_{\alpha}\right)\right\rangle$, from (2.2) and (3) we obtain (6). Lastly, (6) implies (7). 
(3.2) Let $\left(\alpha, \alpha^{\prime}\right)$ be a critical pair. Then the following are true.

(1) $1 \neq\left[Z_{\alpha}, Z_{\alpha^{\prime}}\right] \subseteq Z_{\alpha} \cap Z_{\alpha^{\prime}}$.

(2) $\left(\alpha^{\prime}, \alpha\right)$ is also a critical pair.

(3) $Z_{\alpha} / C_{Z_{\alpha}}\left(G_{\alpha}\right)$ is an irreducible $\operatorname{FF~} \mathrm{GF}(p) \bar{G}_{\alpha}$-module where $\bar{Z}_{\alpha^{\prime}}$ is a quadratic offending subgroup of $\bar{G}_{\alpha}$.

PROOF. (1) and (2) are given by (3.1.7). Now note that, by symmetry, we may assume

$$
\left|Z_{\alpha} / Z_{\alpha} \cap O_{p}\left(G_{\alpha^{\prime}}\right)\right| \leq\left|Z_{\alpha^{\prime}} / Z_{\alpha^{\prime}} \cap O_{p}\left(G_{\alpha}\right)\right|
$$

By (3.1.6), this says that $\bar{Z}_{\alpha^{\prime}}$ is an offending subgroup of $\bar{G}_{\alpha}$ acting on $Z_{\alpha}$. Therefore, it follows from hypothesis (A.1), (2.3), and (3.2.1) that (3) holds and that we have equality in (*). Hence (3) is true with $\alpha$ and $\alpha^{\prime}$ switched.

(3.3) $b \leq 4$.

PROOF. Let us suppose $b \geq 6$. In the context of our proof, whenever $\bar{G}_{\delta}, \delta \equiv \alpha$, has an unambiguous offending subgroup implicitly given by (3.2.3) we take $g_{\delta}$ to be an element of $G_{\delta}$ which gives the generational property in hypothesis (A.3). We make our argument in several steps.

(1) Let $\left(\alpha, \alpha^{\prime}\right)$ be a critical pair and let $\alpha-1=\alpha+1^{g_{\alpha}}$. If $\delta \in \Delta(\alpha-1)$ such that $\left(\delta, \alpha^{\prime}-2\right)$ is not a critical pair, then $Z_{\delta} Z_{\alpha} \unlhd G_{\alpha}$.

Since $b \geq 6$, it follows from (3.1) that $Z_{\delta} Z_{\alpha}$ act quadratically on $Z_{\alpha^{\prime}}$. Hence by (3.2) and hypothesis (A.2), we get that $Z_{\delta} Z_{\alpha} O_{p}\left(G_{\alpha^{\prime}}\right)=Z_{\alpha} O_{p}\left(G_{\alpha^{\prime}}\right)$. We then observe that this means

$$
Z_{\delta} Z_{\alpha} \unlhd\left\langle Z_{\alpha^{\prime}}, S_{\alpha-1}\right\rangle .
$$

The latter group by hypothesis (A.3) is $G_{\alpha}$.

(2) There exists a half-line $l=\left(\ldots, \alpha-2, \alpha-1, \alpha, \ldots, \alpha^{\prime}\right)$ such that for each $i \in \mathbf{Z},(\alpha+2 i, \alpha+2 i+b) \subseteq l$ is a critical pair and for each $\delta \in l$ with $\delta \equiv \alpha$ and $\delta-2, \delta+2 \in l$, we have $\delta+2^{g_{\delta}}=\delta-2$.

Since $\left\langle Z_{\delta}: \delta \in \Delta(\alpha-1)\right\rangle \nsupseteq G_{\alpha},(1)$ immediately yields through induction a half-line $l_{1}$ with the right amount of critical pairs, and such that for all $\mu \in l_{1}$ with $\mu \equiv \alpha, \mu+1^{g_{\mu}}=\mu-1$ where $\mu+1, \mu-1 \in l_{1}$. Now take any critical pair $\left(\lambda, \lambda^{\prime}\right)$ in $l_{1}$ and let $\xi=\lambda+2^{g_{\lambda}}$. We claim that $\left(\xi, \lambda^{\prime}-2\right)$ is also a critical pair. Suppose this is false. Then (1) says that

$$
Z_{\xi} Z_{\lambda}=Z_{\lambda+2} Z_{\lambda}
$$

But this means that $Z_{\lambda-b+2}$ centralizes $Z_{\lambda+2} Z_{\lambda}$, which contradicts the fact that $(\lambda-b+2, \lambda+2)$ is a critical pair. Therefore we may construct $l_{1}$ so that it passes through $\xi$ and hence by induction we obtain the existence of the half-line $l$.

(3) There is no critical pair $\left(\lambda, \lambda^{\prime}\right)$ in $l$ such that $\bar{Z}_{\lambda^{\prime}} \unlhd \bar{S}_{\lambda+1}$ in $\bar{G}_{\lambda}$.

Assume $\left(\lambda, \lambda^{\prime}\right)$ has the above normality property. Then we note that

$$
R=\left[Z_{\lambda}, Z_{\lambda^{\prime}}\right]=\left[Z_{\lambda}, Z_{\lambda^{\prime}} O_{p}\left(G_{\lambda}\right)\right] \unlhd S_{\lambda+1} .
$$

Thus as a consequence of hypothesis (A.3), (2), and $b \geq 6$, we get that

$$
R \unlhd\left\langle Z_{\lambda^{\prime}+2}, S_{\lambda+1}\right\rangle=G_{\lambda+2},
$$

and hence also that $R \unlhd\left\langle Z_{\lambda^{\prime}+4}, S_{\lambda+3}\right\rangle=G_{\lambda+4}$. But this means $R \unlhd G_{\lambda}$, which is clearly impossible. 
(4) The final contradiction.

Take an arbitrary critical pair $\left(\lambda, \lambda^{\prime}\right)$ in $l$ and again set $R=\left[Z_{\lambda}, Z_{\lambda^{\prime}}\right]$. Since in $\bar{G}_{\lambda+2}, \bar{Z}_{\lambda^{\prime}+2} \notin \bar{X}_{\lambda+3}$, it follows from hypothesis (A.3) and $b \geq 4$ that

$$
X_{\lambda+2}=\left\langle Z_{\lambda^{\prime}+2},\left\langle Z_{\lambda^{\prime}+2}^{g_{\lambda+2} S_{\lambda+1}}\right\rangle\right\rangle
$$

centralizes $R$ and acts transitively on $\Delta(\lambda+2)$. For the exact same reason,

$$
X_{\lambda+4}=\left\langle Z_{\lambda^{\prime}+4},\left\langle Z_{\lambda^{\prime}+4}^{g_{\lambda+4}} S_{\lambda+3}\right\rangle\right\rangle
$$

is transitive on $\Delta(\lambda+4)$. Now we observe that as $X_{\lambda+2}$ is transitive on $\Delta(\lambda+2)$ and $b \geq 6$, we also know that $X_{\lambda+4}$ centralizes $R$.

Since $g_{\lambda+2} \in X_{\lambda+2} S_{\lambda+1}$, there exists some $x \in X_{\lambda+2}$ such that $\lambda+4^{x}=\lambda$. Therefore, we conclude that $R$ is centralized by some $X_{\lambda}$ in $G_{\lambda}$ that acts transitively on $\Delta(\alpha)$. This clearly violates $(2.4)$.

The proof of our theorem is now complete.

\section{REFERENCES}

[B] B. Baumann, Über endliche Gruppen mit einer zu $L_{2}\left(2^{n}\right)$ isomorphen Faktorgruppe, Proc. Amer. Math. Soc. 74 (1979), 215-222.

[C] N. Campbell, Pushing up in finite groups, Ph.D. thesis, California Inst. of Technology, 1979.

[G] D. Goldschmidt, Automorphisms of trivalent graphs, Ann. of Math. (2) 111 (1980), 377-406.

[Gn] D. Gorenstein, Finite simple groups, Plenum Press, New York and London, 1982.

[M] U. Meierfrankenfeld, Pushing up $\operatorname{Sp}(4, q)$, preprint.

[N] R. Niles, Pushing-up in finite groups, J. Algebra 57 (1979), 26-63.

[S] B. Stellmacher, Pushing up, Arch. Math. 46 (1986), 8-17.

[T] F. Timmesfeld, Amalgams with rank 2 groups of Lie type in char 2, preprint.

FAKULTät FÜR MATHEMATIK, UNIVERSität BIELEFELD, UNIVERSitätSSTRASSE, 4800 BIELEFELD (FRG)

Current address: Department of Mathematics, University of Arizona, Tucson, Arizona 85721 